Rigidity, conformation, and solvation of native and oxidized tannin macromolecules in water-ethanol solution

Dražen Zanchi, Petr V. Konarev, Christophe Tribet, Alain Baron, Dmitri I. Svergun, and Sylvain Guyot

Citation: J. Chem. Phys. 130, 245103 (2009); doi: 10.1063/1.3156020

View online: https://doi.org/10.1063/1.3156020

View Table of Contents: http://aip.scitation.org/toc/jcp/130/24

Published by the American Institute of Physics 


\title{
Rigidity, conformation, and solvation of native and oxidized tannin macromolecules in water-ethanol solution
}

\author{
Dražen Zanchi, ${ }^{1, a)}$ Petr V. Konarev, ${ }^{2}$ Christophe Tribet, ${ }^{3}$ Alain Baron, ${ }^{4}$ Dmitri I. Svergun, ${ }^{2}$ \\ and Sylvain Guyot ${ }^{4, a)}$ \\ ${ }^{1}$ Laboratoire de Physique Théorique et Hautes Energies, 4 place Jussieu, BP 126, \\ 75252 Paris CEDEX 05, France \\ ${ }^{2}$ European Molecular Biology Laboratory, EMBL clo DESY, Notkestrasse 85, \\ D-22603 Hamburg, Germany and Institute of Crystallography, Russian Academy of Sciences, \\ Leninsky pr. 59, 117333 Moscow, Russia \\ ${ }^{3}$ Laboratoire de Physico-Chimie des Polymères et Milieux Dispersés, ESPCI ParisTech, \\ UPMC et CNRS UMR 7615, 10 rue Vauquelin, 75231 Paris CEDEX 05, France \\ ${ }^{4}$ UR117 Cidricoles et Biotransformation des Fruits et Légumes, INRA, F35650 Le Rheu, France
}

(Received 3 February 2009; accepted 21 May 2009; published online 30 June 2009)

\begin{abstract}
We studied by light scattering and small angle x-rays scattering (SAXS) conformations and solvation of plant tannins (oligomers and polymers) in mixed water-ethanol solutions. Their structures are not simple linear chains but contain about $6 \%$ of branching. Ab initio reconstruction reveals that monomers within a branch are closely bound pairwise. The chains are rather rigid, with the Kuhn length $b=13 \pm 3 \mathrm{~nm}$, corresponding to about 35 linearly bound monomers. Contribution of solvation layer to SAXS intensity varies in a nonmonotonic way with ethanol content $\phi_{A}$, which is an indication of amphipathic nature of tannin molecules. Best solvent composition $\phi_{A}^{B}$ is a decreasing function of polymerization degree $N$, in agreement with increasing water solubility of tannins with $N$. Polymers longer than $b$ present a power-law behavior $I \sim Q^{-d}$ in the SAXS profile at high momentum transfer $Q$. The monotonic decrease in $d$ with increasing $\phi_{A}$ (from 2.4 in water to 1.9 in ethanol) points that the tannins are more compact in water than in ethanol, presumably due to attractive intramolecular interactions in water. Tannins were then oxidized in controlled conditions similar to real biological or food systems. Oxidation does not produce any intermolecular condensation, but generates additional intramolecular links. Some oxidation products are insoluble in water rich solvent. For that reason, we identify these species as a fraction of natural tannins called "T1" in the notation of Zanchi et al. [Langmuir 23, 9949 (2007)]. Within the fraction left soluble after oxidation, conformations of polymeric tannins, despite their higher rigidity, remain sensitive to solvent composition. (C) 2009 American Institute of Physics. [DOI: 10.1063/1.3156020]
\end{abstract}

\section{INTRODUCTION}

Biological task of tannins is to protect plants from virus, fungi, bacteria, and higher herbivores. ${ }^{1}$ Mechanisms of this protection are based on essentially physical interactions of tannins with predator's proteins. For example, tannins inhibit the cell-wall lyzing enzymes secreted by invading fungi and bacteria, ${ }^{2}$ induce aggregation of salivary proteins of higher herbivores thus producing astringency, ${ }^{3}$ and inhibit digestive enzymes. ${ }^{4}$ The high affinity for proteins and strong antioxidant activity makes tannins important in food technology, pharmaceutics, and cosmetics. For instance, recently, it has been shown that a simple tannin EGCG acts as molecular chaperone for preventing amyloidogenesis. ${ }^{5}$ Also, tannins have been recently proposed as site specific tools for nanomanipulaton of proteins. ${ }^{6}$ Therefore the use of tannins for controlling pathways of protein folding and aggregation can be envisaged. The practical importance of tannins contrasts with the limited level of structural characterization available to date. At length scale above the size of a monomer, the

\footnotetext{
${ }^{a)}$ Authors to whom correspondence should be addressed. Electronic mail: drazen.zanchi@lpthe.jussieu.fr and sylvain.guyot@rennes.inra.fr.
}

complexity of these macromolecules and their propensity to form aggregates make it difficult to analyze their conformation properties, specifically because of complicated chemical structures (branching, oxidation) and solvation properties (possible networks of aromatic stacking, hydrogen bonds, Coulombic interactions). ${ }^{7}$

Tannins in fruits are oligomeric and polymeric catechins (i.e., procyanidins, also called condensed tannins) with a typical structure shown in Fig. 1. They are weak acids, with $p \mathrm{~K}_{a}$ between 9 and 11, which means that in neutral-to-acid $p \mathrm{H}$ they are not charged. However, they can acquire some charge via complexation with organic acids. ${ }^{7}$ Tannins binding to proteins are dominated by hydrophobic stacking and hydrogen bond attractions between catechol functions of tannins (B in Fig. 1) and proline residues of proteins. Both degree of polymerization $(N)$ and molecular structure of some tannin determine its affinity for a particular protein. Roughly speaking, the longer the tannin polymer and the higher its pyrogallol content, the higher is its power to induce aggregation and precipitation of proteins. ${ }^{8}$

Another aspect of tannin's biological role is their fast (mostly enzymatic) oxidation, e.g., browning of apple and 


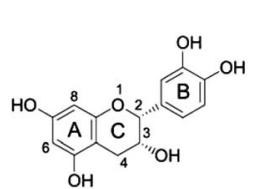

(-)-epicatechin

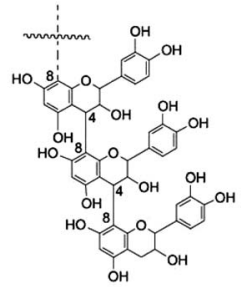

Linear tannin

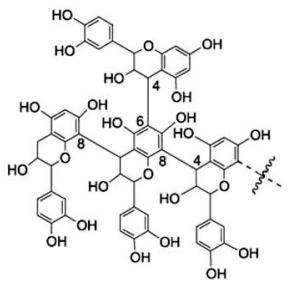

Branched tannin
FIG. 1. Structure of tannins studied in this work. The monomer is (-) epicatechin made of two aromatic rings (a) and (b) and a pyrane ring (c). Monomers are linked through $\mathrm{C} 4-\mathrm{C} 8$ and $\mathrm{C} 4-\mathrm{C} 6$ interflavanol bonds. Combination of two bonds includes ramifications in polymer.

cider, fermentation of tea leafs, making of red wine, etc. ${ }^{9}$ The oxidation of fruit tannins have two main easily observable consequences: Increase in tanning activity ${ }^{10}$ and insolubilization in water solvent. Both effects are usually referred to as an increase in effective hydrophobicity of tannins upon oxidation. However, the questions still make debate on whether tannins become more hydrophobic upon oxidation and what differs from soluble and insoluble tannin molecules.

It is well known that solubility of fruit tannins increases if ethanol is added to water solvent. This effect is well known in, e.g., wine making industry, where tannins are better extracted from grape skins when must is richer in ethanol. In a recent study of native grape tannins, ${ }^{7}$ two main tannin populations T1 and T2 were identified within a single tannin fraction obtained by fractional elution. In solvent with dominant water content, T2, the highly soluble tannin was clearly distinguished from T1, the poorly soluble one, while at high ethanol content both fractions were highly soluble. At intermediate solvent compositions, T1 tannins formed metastable colloidal particles, stabilized by anionic surface charges, presumably attached to tannins by complexation with organic acids. The attractive interactions between $\mathrm{T} 1$ tannins are found to be of hydrophobic nature. T1 fraction remains mysterious for its ill-defined chromatographic behavior and related impossibility to analyze. It was assumed that T1 tannins are a subpopulation of oxidized tannins. One of the goals of the present paper is to confirm this conjecture.

In the present study, we investigate the size and structure in solution of the native and oxidized tannins as polymeric species with solvent dependent interactions. We used several methods to study the geometrical and physical properties of tannin molecules and analyze their shape, measure their molecular mass, their radius of gyration, and the self-similar scaling dimension. We also analyzed the width and the shape of solvation layer as a function of solvent composition (ethanol/water fraction $\phi_{A}$ ) and of tannin degree of polymerization $N$. Basically, we address the questions of variation with solvant quality of the conformation and aggregation of oligomeric to polymeric tannins, which should bring better understanding of the relationships between the molecular solvation in water-ethanol and intra-/intertannins interactions at the scale of the macromolecules. The consequences of oxidation are similarly considered from the point of view of solvation effects.

We concentrated on homopolymers of (-)-epicatechin, as representatives of "fresh," nonoxidized tannins shown on Fig. 1. We analyzed macromolecules from monomer to polymers with polymerization degree up to 223 . Dominant interflavane bonds are $\mathrm{C} 4-\mathrm{C} 8$, with minority of $\mathrm{C} 4-\mathrm{C} 6$ bonds, that may form a junction for ramification in the polymer chain. ${ }^{11,12}$ Polymerized epicatechins can rotate around the $\mathrm{C} 4-\mathrm{C} 8$ and $\mathrm{C} 4-\mathrm{C} 6$ bonds. In the case of the B2 dimer (C4-C8 linked) two free energy minima with respect to this rotation were found, corresponding to one "open" and one "closed" conformation, with an activation barrier for their transconversion of $15-20 \mathrm{kcal} / \mathrm{mol}$ (i.e., $\left.20-30 k_{B} T\right){ }^{13} \mathrm{It}$ is expected that in good solvent (ethanol), the open conformation is populated while in poor solvent (water), the closed conformation dominates. The situation is less simple for higher oligomers and polymers and much more complicated in the case of oxidized tannins. In fact, even native, nonoxidized polyphenol molecules interact with solvent in a poorly understood way. Some indications of preferential solvation were reported in Ref. 7: The solvent in contact with the tannin does not have the same composition as the bulk solvent. However, details of the structure, composition, and width of solvation layer are unknown. The solvation layer is presumably responsible for the intrachain interactions that determine the free energy profile and, consequently, the conformation of the polymer. Thus, we expect that the solvation layer and conformation are related. To summarize, we are looking for solvent induced changes of fresh and oxidized tannins. We expect that more "unfolded" and extended structures will form in good solvent and more compact conformations will be found in poor solvent.

\section{MATERIALS}

\section{A. Preparation of native oligomeric and polymeric procyanidin fractions}

Apple oligomeric procyanidin fractions from monomer to heptamer were obtained from cider apple materials (Kermerrien variety), using successive solvent extractions followed by a fractionation on semipreparative normal phase HPLC, to yield almost monodisperse fractions in terms of degree of polymerization, as described by Guyot et al. ${ }^{14}$ Chromatogram shown in Fig. 2(a) displays well resolved oligomers ranging from DP2 to DP7.

Four somewhat polydisperse fractions of procyanidin polymers were obtained from the Avrolles cider apple variety. The resulting fractions yielded tannins with polydispersity between 1.2 and 2 (i.e., the ratio of weight-average to number-average molar mass) and were called accordingly to their number-averaged degree of polymerization DP28, DP75, DP150, and DP223. Successive solvent extractions of the apple powder were performed as already described. Then, the aqueous acetone extract containing polymers was fractionated by HPLC at the preparative scale by using a particular fractionation procedure for which C18 stationary phase was saturated by the phenolic molecules. ${ }^{15}$

\section{B. Preparation of oxidized procyanidin fractions}

(-)-Epicatechin, native purified procyanidin fractions ranging from DP2 to DP7 and polymeric fraction DP28 were 

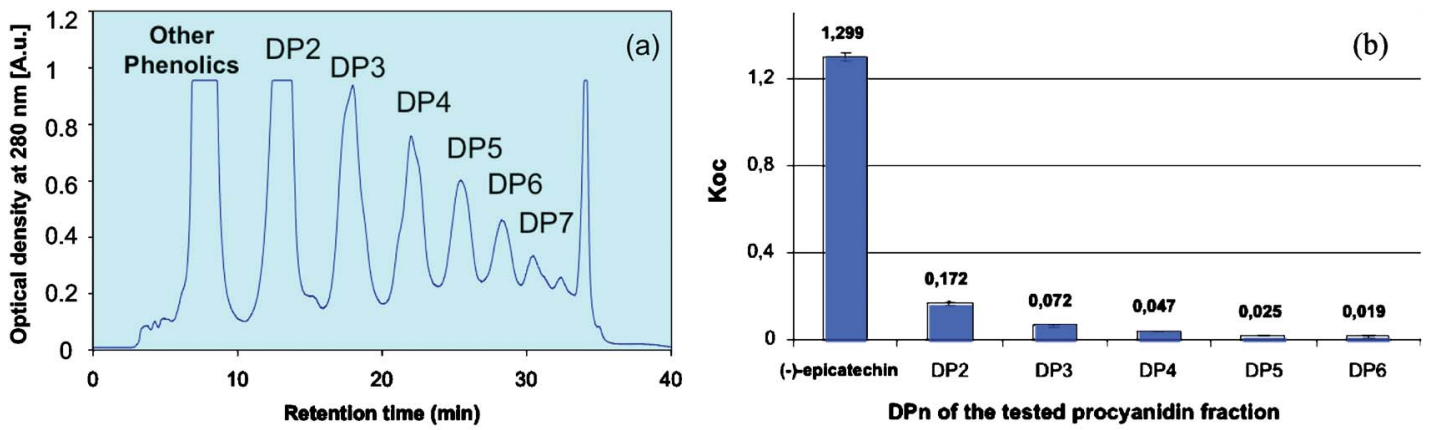

FIG. 2. (Color online) Some characteristics of oligomeric tannins used in this study. (a) Chromatogram from semipreparative HPLC fractionation (detection by absorption at $280 \mathrm{~nm}$ ) resolving oligomers from DP2 to DP7, (adapted from Ref. 14). (b) Octanol-water partition coefficient for tannin oligomers from DP1 (epicatechin) to DP6.

chemically oxidized by periodate immobilized on a solid matrix according to a method of Le Bourvellec et al. ${ }^{16}$ Each fraction $(2 \mathrm{mM}$ in epicatechin equivalent, or $0.58 \mathrm{~g} / \mathrm{l}$ ) in pure anhydrous methanol $(20 \mathrm{ml})$ was stirred in the presence of the periodate form of amberlite GC400 type I ( $2 \mathrm{mM})$ for 10 $\min$ at $30{ }^{\circ} \mathrm{C}$. Then, the mixtures were immediately filtered (0.45 $\mu \mathrm{m}, \mathrm{PTFE})$ to remove the resin and the filtrates were incubated for $2 \mathrm{~h}$ at $30{ }^{\circ} \mathrm{C}$. Residual polyphenol quinoid forms were reduced by adding $20 \mathrm{ml}$ of an aqueous sodium thiosulfate $(6 \mathrm{mM})$. After removing methanol by evaporation under vacuum, aqueous oxidized procyanidins fractions were purified by solid phase extraction on C18 SepPak cartridges to remove thiosulfate salts. ${ }^{14}$ Finally, freeze-dried oxidized fractions were obtained as amorphous powders.

\section{Characterization of native and oxidized procyanidin fractions}

In order to determine (i) the percentage of procyanidin structures, (ii) the nature of constitutive catechin units, and (iii) the average degree of polymerization, all fractions were analyzed by thiolysis associated with reversed phase HPLC for the characterization of the procyanidins. ${ }^{14}$ All unoxidized fractions contained about $90 \%$ of native procyanidins with (-)-epicatechin units accounting for more than $95 \%$ of the total units. The measured number average polymerization degree $N$ of DP2, DP3, DP4, DP5, DP6, and DP7 fractions were $2.0,3.0,3.8,4.8,5.6$, and 6.3 , respectively.

The amphypatic properties of DP fractions were studied by partition between octanol and water. In $1.5 \mathrm{ml}$ vials, $2 \mathrm{mg}$ of oligomeric fractions (from DP1 to DP6) were dissolved in $0.5 \mathrm{ml}$ of purified water and $0.5 \mathrm{ml}$ of n-octanol were added. The mixture was vortexed for one minute and centrifugated for $10 \mathrm{~min}$ at $10000 \mathrm{rpm}$. After freeze drying of the water phases and evaporation of octanol, tannins were quantified in the two phases by thiolysis and reversed phase HPLC. ${ }^{14}$ The partition coefficient $K_{o c}$ (ratio of the concentration in octanol over concentration in water), shown in Fig. 2(b), indicates that the solubility in water increases with increasing molecular weight.

Analysis of oxidized fractions showed that the percentage of native procyanidin structures (as estimated by thiolysis) decreased in the $20 \%-40 \%$ range, indicating that procyanidin structures have been extensively altered by oxidation, leading to the formation of new oxidative linkages which are resistant to thiolytic degradation. Native and oxidized fractions were also analyzed by liquid chromatography coupled to electrospray-ion trap mass spectrometry in the negative mode (LC-MS) in order to characterize the main molecular species. Conditions were similar to those previously used for the characterization of some phenolic oxidation products in cider samples, ${ }^{17}$ except for the electrospray parameters, which were optimized on the pseudomolecular ions $[M-H]^{-}$ of tetrameric procyanidins $(m / z=1153)$. The LC-MS data of native oligomers were highly monodisperse: most of the peaks observed on the MS base-peak chromatograms (i.e., chromatograms corresponding to the selective detection of the most intense ion versus retention time) revealed mass spectra in accordance with the expected procyanidin oligomers in the considered fraction. For example, chromatographic peaks of nonoxidized DP3 fraction corresponded to mass per charge ratio $m / z=865$ as most intense ions on the mass spectra, matching the molecular weight of DP3 molecules $(M=866 \mathrm{Da})$.

For oxidized fractions, the MS base-peak chromatograms were much more complex. As expected, in addition to the peaks corresponding to residual native molecules that were not oxidized, many new peaks corresponding to oxidation products were detected. Most of MS peaks corresponded to the native molecule having lost 2, 4, sometimes 6, and even more hydrogen atoms in accordance to the formation of one, two, or three (and even more depending on the polymerization state) new oxidation-induced intramolecular linkages. Other peaks matched with one or two water molecules addition following the quinone formation $(+16$ or +32 mass units compared to the corresponding unoxidized molecule). Finally, a third category of products showed MS data in agreement with addition of one or two iodine atom(s) (128 Da) per epicatechin unit. This reaction may have occurred as a consequence of the presence of free iodine anions generated from periodate anions during oxidation. Interestingly, no MS signal attesting for the formation of intermolecular coupling was detected. We concluded that, contrary to what is often mentioned in literature, oxidation proceeds rather by intramolecular oxidative coupling than by polymerization. The ratio of tannin molecules affected by oxidation was typically more than $80 \%$ for small oligomers $(N=2)$ and we assumed that it is at least as high for higher $N$. 


\section{METHODS}

\section{A. Ultracentrifugation}

After sonication for $15 \mathrm{~min}$, solutions of tannin powders in water $(5 \mathrm{~g} / \mathrm{l}$ and $10 \mathrm{~g} / \mathrm{l})$ were centrifuged in the MLA 80 rotor of a Beckman TLA centrifuge (70 000 rpm, i.e., $250000 \mathrm{~g}$, for $15 \mathrm{~min}$., at $20{ }^{\circ} \mathrm{C}$ ). The absorbance at 280 $\mathrm{nm}$ of the initial samples and supernatants were measured to determine the fraction of soluble tannins.

\section{B. Static and dynamic light scattering}

Light-scattering experiments were performed on a CGS-3 goniometer (ALV GmbH) equipped with a ALV/LSE5003 multiple- $\tau$ digital correlator (ALV GmbH), a $\mathrm{He}-\mathrm{Ne}$ laser $(\lambda=632 \mathrm{~nm})$, and a $\mathrm{C} 25 \mathrm{P}$ circulating water bath (Thermo Haake). The temperature was set at $25^{\circ} \mathrm{C}$. For dynamic light scattering (DLS) analysis, the homodyne intensity-intensity correlation function $G(q, t)$ was related to the autocorrelation function of the scattered field, $g(t)$. Inversion of the function $g(t)$ was performed using the program CONTIN, providing a distribution of apparent Stokes radii, $R_{H}$.

Tannins in solution are expected to be very anisotropic objects, with $R_{H}$ much lower than the end-to-end length. For these reasons, we represented them as prolate ellipsoids characterized by the parameter $p=a / b$, with $a$ and $b$ being the long and short axes. In order to calculate effective $R_{H}$ from known molecular mass or volume $V$, we used Perrin's formula, ${ }^{18}$

$$
R_{H}=R_{s} / F(p),
$$

where $R_{s}$ is the radius of the equivalent sphere with volume $V$ and the function $F(p)$ writes

$$
F(p)=\frac{p^{1 / 3}}{\sqrt{p^{2}-1}} \ln \left(p+\sqrt{p^{2}-1}\right) .
$$

\section{Small angle $x$-rays scattering experiments}

The synchrotron radiation $\mathrm{x}$-ray scattering data were collected on the X33 camera of the European Molecular Biology Laboratory on the storage ring DORIS III (DESY, Hamburg, Germany ${ }^{19}$ ). Solutions of tannins (with polymerization degree from 1 to 150 ) were measured at $25^{\circ} \mathrm{C}$, solute concentrations ranging from 1 to $10 \mathrm{mg} / \mathrm{ml}$ in water-ethanol buffers. The data were recorded using a MAR345 twodimensional image plate detector at a sample-detector distance of $2.7 \mathrm{~m}$ and a wavelength of $\lambda=0.15 \mathrm{~nm}$, covering the range of momentum transfer $0.05<Q<5 \mathrm{~nm}^{-1}(Q$ $=4 \pi \sin \theta / \lambda$, where $2 \theta$ is the scattering angle). To check for radiation damage, the data were collected in two $2-\mathrm{min}$ exposures. These measurements indicated no changes in the scattering patterns with time, demonstrating no measurable radiation damage. The data were averaged after normalization to the intensity of the incident beam and the scattering of the buffer was subtracted. All data manipulations were performed using the program package PRIMUS. ${ }^{20}$

The forward scattering $I(0)$ and the radius of gyration $\left(R_{g}\right)$ were evaluated using the Guinier approximation assum- ing that at very small angles $\left(Q \ll 1 / R_{g}\right)$ the intensity is represented as $I(Q)=I(0) \exp \left(-(1 / 3)\left(Q R_{g}\right)^{2}\right)$. These quantities and the maximum diameter $D_{0}$ of the particles were also computed from the entire scattering pattern using the program GNOM. ${ }^{21}$ The excluded volume, $V_{P}$, of the particle was computed from the Porod invariant. ${ }^{22}$

Small angle X-rays scattering (SAXS) profiles of tannin solutions were also fitted to the scattering function $F_{\sigma}^{d}\left(R_{g} Q\right)$, which writes

$$
F_{\sigma}^{d}\left(R_{g} Q\right)=\int_{0}^{\infty} \xi_{d}\left(R_{g} Q x\right) f_{\sigma}(x) d x,
$$

with

$$
\xi_{d}(t)=\left(1+\frac{t^{2}}{3 d / 2}\right)^{-d / 2}
$$

being the Fisher-Burford ${ }^{23}$ function. For $t=z Q$ this function is a form factor for self-similar structures with correlation length $z$ and fractal dimension $d$. In the case of noninteracting particles with bushy structure, $z$ is simply the radius of gyration $R_{g}$, while the dimension $d$ measures the compactness of the structure. Polydispersity is included into the scattering function by the log-normal distribution with the width $\sigma$, which writes

$$
f_{\sigma}(x)=\frac{1}{\sqrt{2 \pi} \sigma} \exp \left[-\frac{1}{2}\left(\frac{\ln x}{\sigma}\right)^{2}\right] .
$$

From the fits, we obtained the average radius of gyration $R_{g}$ of the particles, the width $\sigma$ of their size distribution, and the dimension $d$ that characterized their internal structure and/or the surface roughness.

The particle shape was reconstructed, $a b$ initio, using the program DAMMIN, ${ }^{24}$ which represents the particle as a collection of $M_{b} \gg 1$ densely packed beads inside a sphere with the diameter $D_{0}$. Each bead belongs either to the particle or to the solvent and the shape is described by a binary string of length $M_{b}$. Starting from a random string, simulated annealing is employed to search for a compact model that fits the experimental data $I_{\exp }(Q)$ to minimize the discrepancy $\chi$,

$$
\chi^{2}=\frac{1}{n-1} \sum_{j}\left[\frac{I_{\mathrm{exp}}\left(Q_{j}\right)-c I_{\mathrm{calc}}\left(Q_{j}\right)}{\delta\left(Q_{j}\right)}\right]^{2},
$$

where $n$ is the number of experimental points, $c$ is a scaling factor, $I_{\exp }\left(Q_{j}\right)$ and $I_{\text {calc }}\left(Q_{j}\right)$ are the experimentally determined and calculated intensities, respectively, and $\delta\left(Q_{j}\right)$ is the experimental error at the momentum transfer $Q_{j}$. The program GASBOR (Ref. 25) was subsequently used to create $a b$ initio models consisting of dummy residues instead of beads. In this program simulated annealing is employed to construct a model with a "chainlike" distribution of beads that provides the best fit to the experimental data. For $a b$ initio analysis, multiple runs of both DAMMIN and GASBOR were performed to verify the stability of the solution and typical three-dimensional (3D) reconstructions are presented in Figs. 11 and 14. 


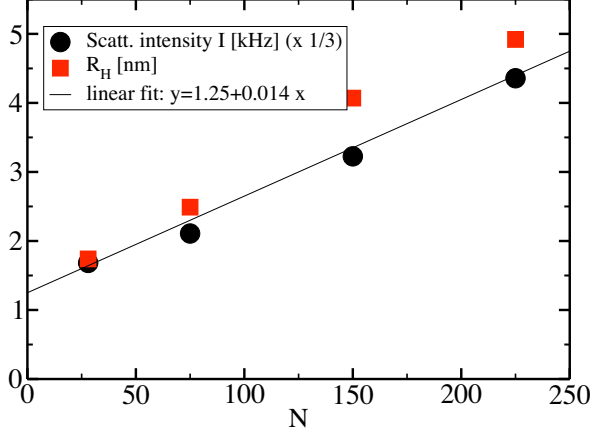

FIG. 3. (Color online) Results of the DLS experiment on tannin polymers, 5 $\mathrm{g} / \mathrm{l}$, in water: average scattering intensity and hydrodynamic radius as functions of tannin polymerization degree $N$.

\section{RESULTS}

\section{A. Native tannins}

First we explored the tannin polymers extracted from apples with number average polymerization from $N=28$ to 223. HPLC experiments showed that samples are to more than $90 \%$ pure water soluble procyanidines. This fact was confirmed by ultracentrifugation of samples in water; we typically pelleted very low fraction (less than 4\%-5\%) of insoluble molecules $\mathrm{T} 1$. The molecules remaining in supernatant were more than $95 \%$ native polyepicatechins.

We started by measuring average scattered light intensity and hydrodynamic radii of tannin polymers by DLS. Results obtained for tannin in water at $5 \mathrm{~g} / \mathrm{l}$ are shown on Fig. 3. Scattered intensity (measured in number of photons per second $(\mathrm{kHz})$ at a laser power of $0.1 \mathrm{~W})$ increases linearly with $N$, as expected for individually solvated molecules. The finite value of intensity extrapolated to $N=0$ is in fact the background scattering from just water. Hydrodynamic radius also increases linearly with $N$. This is an indication that the scatterers are not simply compact and globular because in that case hydrodynamic radius would scale as $N^{1 / 3}$. On the contrary, data can be well understood if we assume that the anisotropy of scatterers increases with $N$. For this purpose, we approximated the shape of tannin polymers by prolate ellipsoids with the length $2 a$ and width $2 b=2 a / p$. We assumed that molecules do not form aggregates so that their volume is $\nu=N \nu_{0}$, where $\nu_{0}=0.3 \times 0.8 \times 0.8 \mathrm{~nm}^{3}$ is the known volume of one monomer (epicatechin). The ratio $p$ is obtained from $R_{H} / R_{s}$ ratio using the formula (1) and calculating the equivalent compact sphere radius $R_{S}$ from the molecular volume $\nu$ as $R_{s}=(3 \nu /(4 \pi))^{1 / 3}$. Results are shown on Fig. 4. The molecules diffuse as strongly anisotropic rodlike objects, with $p=b / a$ varying from 12 for 28-mer to about 27 for 223-mer. The overall length of the $N=223$ polymer is as high as $40 \mathrm{~nm}$. These somewhat high values should be taken with caution because this model assumes rigid and compact structures, which are not really the case.

In order to investigate in more detail the conformations of tannins in water, we turned to SAXS experiments. Figure 5 shows the scattering profiles from small oligomeric tannins at $10 \mathrm{~g} / \mathrm{l}$ in water. All curves contain clearly visible form factor of nanometric species with a well defined Guinier regime and corresponding decay over 1-2 decades of intensity.

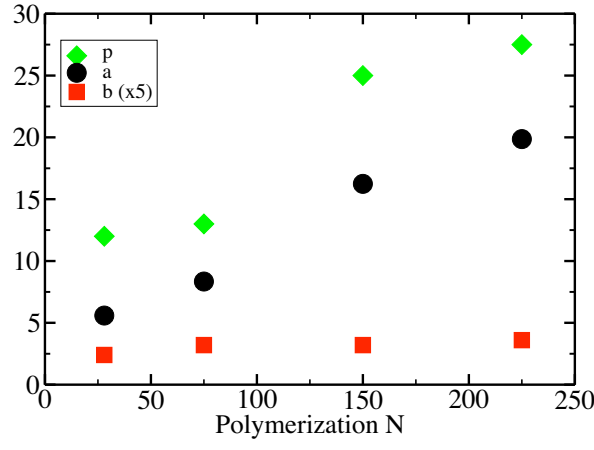

FIG. 4. (Color online) Parameters obtained from our DLS results assuming that tannin molecules diffuse like prolate ellipsoids with long semiaxis $a$, short semiaxis $b$, and ratio $p=a / b$.

At small values of $Q$, there is an up turn of all scattering curves. It is known that this increase in scattering intensity is a contribution of a very small fraction of aggregated tannins and that it is not related to attractive interactions between dissolved small species. ${ }^{7}$ Namely, any native tannin sample contains at least some large aggregates that remain in suspension even after centrifugation. However, their fraction in total sample is negligible: In the present case less than $1 \%$, as found by ultracentrifugation. The fraction of insoluble/ colloidal tannins can, however, become much larger in oxidized samples, as it will be shown in the next section. Forward scattering intensity for small (dissolved) species increases proportionally with $N$, starting from monomer (epicatechin) to DP7. Furthermore, the scattering profile is independent of tannin concentration. These are indications of the absence of intermolecular association and that the scatterers are indeed isolated molecules. Data were fitted to the FisherBurford scattering formula for compact objects $(d=3)$ with radius of gyration $R_{g}{ }^{23}$ These simple fits give us only the very first hints about the scatterers, while more detailed structural analysis will be given in Sec. V.

The SAXS intensities from water solutions of tannins with varying polymerization degree $N=28$ up to 150 are shown in Fig. 6, also displaying the Fisher-Burford fits, which yield gyration radii from low- $Q$ range and power-law regime $I \sim Q^{-d}$ at larger $Q$. This signifies that at scales be-

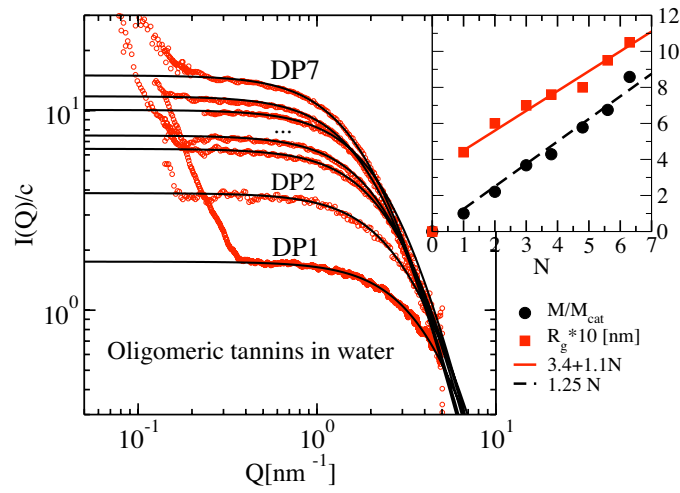

FIG. 5. (Color online) Scattering profiles of native tannins from monomer (epicatechin) to DP7 $(N=6.3)$ in water. From normalized forward scattering intensity $I(Q \rightarrow 0) / c$ we extract the molecular mass. From fits to the FisherBurford scattering function (Ref. 23), we determine the radius of gyration $R_{g}$. 


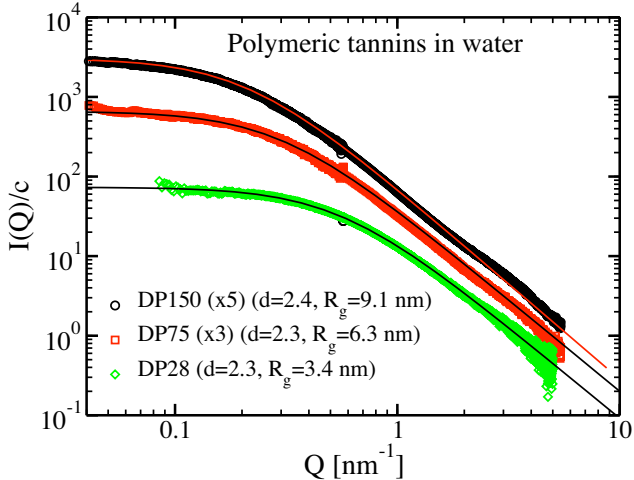

FIG. 6. (Color online) SAXS profiles from native tannin polymers in water at $c=5 \mathrm{mg} / \mathrm{ml}$. We determine the radius of gyration $R_{g}$ and scaling exponent $d$ for $I \sim Q^{-d}$ from fits to the Fisher-Burford scattering function (Ref. 23). Weight averaged degree of polymerization is proportional to normalized forward scattering intensity $I(Q \rightarrow 0) / c$.

tween the monomer size (about $0.8 \mathrm{~nm}$ ) and the molecule's overall size there exist a self-similar regime. For tannins in water, the power law scales with $d$ between 2.3 and 2.4. Values of $d$ higher than 2 indicate either the presence of ramifications (branching) or a contribution of loops or specific attractions between monomers placed far from one another in the chain. Another indication that the tannins behave like branched polymers is that $R_{g} / N^{1 / d}=\operatorname{const}(N)=0.8 \mathrm{~nm}$.

The conformation of macromolecules and the dependence of their size with $N$ depend on the Kuhn length $b$ (twice the persistence length). This length accounts for the interactions between monomers. It is interpreted as rigidity of the chain: oligomers shorter than $b$ should display an elongated rodlike shape. Assuming that tannins are uncharged, the interactions between monomers should depend mainly on the degree and tightness of solvation and possibly on hydrogen bond formation. In fact, tannins are typically much more soluble in ethanol than in water. For that reason, we expect that the interaction between tannins monomers depends on ethanol content in the solvent. In order to get direct evidence of the length scales at which monomer-monomer correlations occur, we addressed the question of rigidity and solvation effects by studying oligomeric and polymeric tannins.

A typical evolution of oligomers' SAXS profile with eth-

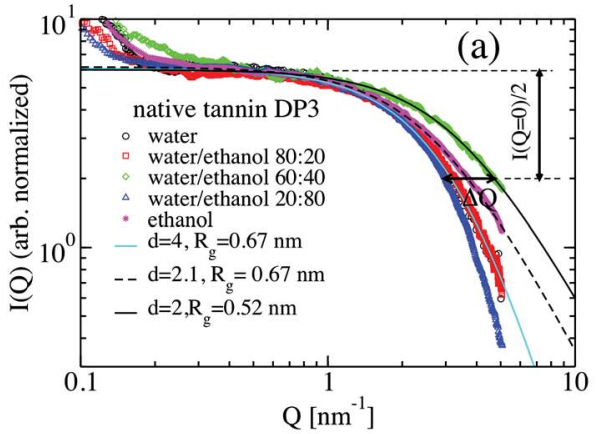

anol volume fraction $\phi_{A}$ is shown in Fig. 7 for tannin trimer $(N=3)$. The apparent size of tannin reaches its smallest value at $\phi_{A}=0.4$ and not in pure water as one would naively expected assuming that the molecule tries to hide the hydrophobic parts of its surface from water. The apparent size of the molecule reaches a maximum at $\phi_{A}=0.8$. At this solvent composition, the interface between solvated tannin and bulk solvent is sharp because $d=4$. The results above are indications of a more complex effect than just gradual variation in polymer conformation with solvent composition. Figure 7(b) shows typical magnitude of the solvent-induced changes in the SAXS profiles as a function of the degree of polymerization $N$. In order to quantify the effect, we introduce quantity $\Delta$,

$$
\Delta \equiv \frac{\Delta Q}{Q_{1 / 2}}
$$

where $\Delta Q$, shown by Fig. $7(\mathrm{a})$, is the magnitude of solvent induced variation in $Q_{1 / 2}$, the momentum transfer at which the scattering intensity is equal to one-half of its forward value. We defined the corresponding direct space variation as

$$
\delta=\Delta \times R_{g} .
$$

Interestingly, $\Delta$ is finite even for monomers (DP1) and diminishes relatively to the molecule size. The invariance of $\delta$ with $N$ (within experimental error) indicates that the change in the scattering pattern is dominated by solvation layer and not by variations in the tannin conformations. Therefore, $\delta$ is interpreted as solvation layer thickness. Experimental value of $\delta$ ranges between 1 and $3 \AA$, which corresponds to one or two molecules thick solvation shell.

In the case of higher polymers $(N>28)$, similar solvation effects cause real perturbations of the conformation. Weak variation in the composition in the solvation shell around the monomers triggers large changes of monomermonomers interaction, which in turn markedly affects the conformation of the whole chain. The phenomenon is well visible on the Kratky plot shown in Fig. 8, for the case of $N=150$ tannin polymer. The scaling regime exists over almost a decade of wave numbers. We identify easily the scaling dimension $d$; in water it is 2.4 , which corresponds to a

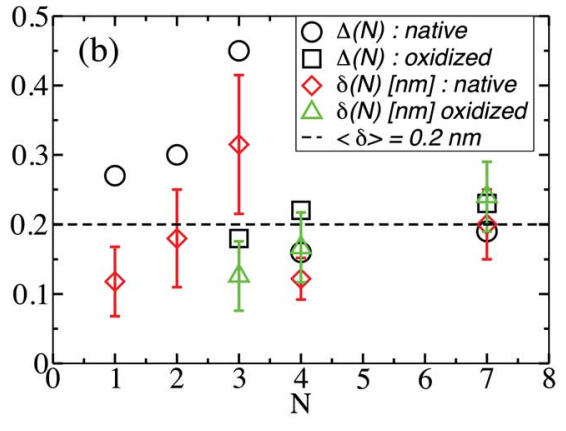

FIG. 7. (Color online) Solvent effect on native tannin trimer $(N=3)$. (a) Scattering profile for several ethanol-water solvent compositions: pure water, $\phi_{A}$ $=20,40,80$ and $100 \%$ ethanol. Data are fitted to the Fisher-Burford scattering function (Ref. 23) by adjusting scaling dimension $d$ and radius of gyration $R_{g}$. The scaling dimension $d$ is here just an indication about the geometry of scatterers. Low values of $d$ indicate more extended or irregular structures, while stronger decay indicates compact objects with sharp surface. (b) Magnitude of solvent effects on the SAXS profiles as a function of polymerizaton number $N$ for native and for oxidized tannins: $\Delta=\Delta Q / Q_{1 / 2}$ and corresponding variation in the size of scatterers $\delta(N)=\Delta(N) \times R_{g}(N)$, corresponding to variations in the solvation layer. 

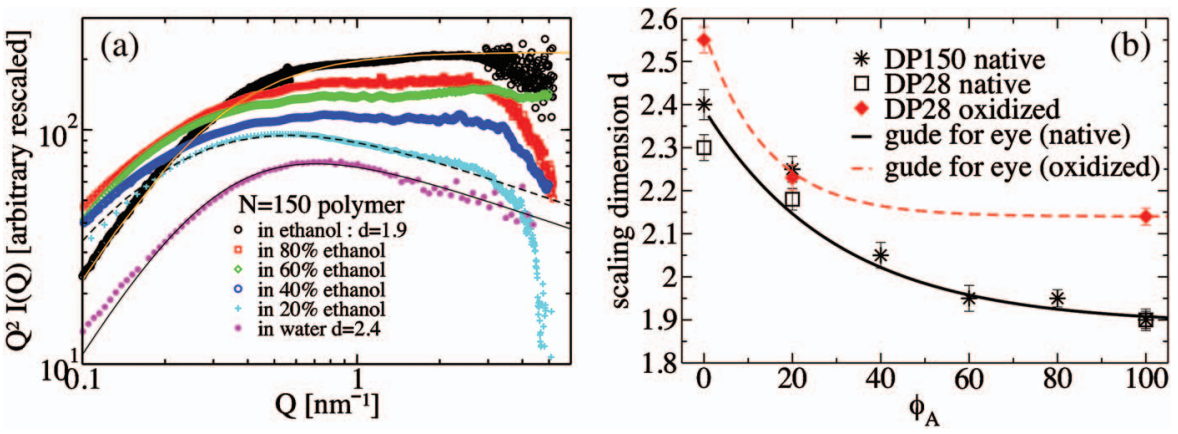

FIG. 8. (Color) (a) SAXS intensities from native tannin polymer $N=150(10 \mathrm{~g} / 1)$ at six ethanol/water fractions. Scattering intensity is multiplied by $Q^{2}$. Power law evolves from in $Q^{-1.9}$ in ethanol to $Q^{-2.4}$ in water. Dimension $d$ is a monotonic function of ethanol fraction $\phi_{A}$. Monomer structure and chain width start to be visible at $Q>3 \mathrm{~nm}^{-1}$, where scattering intensity starts do deviate downward. (b) Dependence $d\left(\phi_{A}\right)$ for native and for oxidized tannins. Dimension $d$ for oxidized tannins varies from 2.55 in water to 2.2 in ethanol, which indicates that molecules adapt to the solvent, but remain more compact than nonoxidized tannin molecules.

rather compact conformation. The value $d$ decreases monotonically as function of ethanol content $\phi_{A}$, reaching finally the value $d=1.9$ in pure ethanol. The fact that $d$ monotonically decreases with increasing $\phi_{A}$ indicates, in agreement with former studies, ${ }^{7}$ that tannin macromolecules interact mostly via hydrophobic attraction. Upon adding ethanol, attractive interactions become weaker and polymer swells.

\section{B. Oxidized tannins}

It was found by LC-MS characterization described in Sec. II that the molecular masses of oxidized tannins differ from the molecular masses of native tannins only by the additional intramolecular bonds. This is a very important result: The oxidation of tannin solution at realistic concentrations (up to about $5 \mathrm{~g} / \mathrm{l}$ ) mostly produces intramolecular condensations. Intermolecular polymerization products were not detected. We expect that the latter can be created in conditions when intermolecular distances become comparable to the intramolecular spacing between monomers. This can happen either at very high concentration or when tannins are in solid state in presence of air and humidity.

An important difference between oxidized and native tannins is that, under their oxidized form, tannins are typically not completely soluble in water-rich solvent $\left(\phi_{A}\right.$ $<50 \%$ ). In order to eliminate nonsoluble species, after mixing and sonication of $10 \mathrm{~g} / \mathrm{l}$ tannin in solvent, all samples were centrifuged at low speed (in Mini-Spin Eppendorf centrifuge, at $13000 \mathrm{rpm}$, for $10 \mathrm{~min}$ ), which left colloidal particles of intermediate size (up to about $500 \mathrm{~nm}$ ) dispersed in the supernatant. For oxidized oligomeric fractions in pure water, the amount of pelleted species varied between about 5 and $60 \mathrm{wt} \%$ of tannin. For instance, for oxidized trimer DP3ox in water, we found $40 \%$ of insoluble tannin. When sample was prepared in $20 \%$ of ethanol-water mixture, almost no pellet was observable after moderate centrifugation, indicating that either all species became soluble or that insoluble fraction remained in colloidal suspension. SAXS experiments show that the second interpretation is correct and give us supplementary information about geometry and interactions of oxidized tannins.

The scattering profiles of both native and oxidized tannin with $N=3$ at low ethanol $\left(\phi_{A}=0\right.$ and $\left.20 \%\right)$ are shown on
Fig. 9. Oxidation enhances scattering intensity at small $Q$, indicating the formation of aggregates. Before measuring SAXS intensities, we eliminated the largest aggregates by mild centrifugation. The water sample contained very large aggregates that were mostly eliminated by centrifugation and some colloidal particles remain in suspension. A stronger increase in the low- $Q$ part of the scattering was observed in $20 \%$ ethanol. Centrifugation of this sample gave no pellet. These facts indicate that the sample contained an important fraction of molecules under the form of metastable colloidal dispersion. The power law decay of the scattering on the colloidal tannins is $Q^{-2.8}$ in this case, but it depends strongly on details of the preparation protocol, as it was already discussed in Ref. 7. At larger values of $Q$ the scattering is due to individual tannin molecules. The radius of gyration is larger for oxidized $\left(R_{g}=0.87 \mathrm{~nm}\right)$ than for native tannins $\left(R_{g}\right.$ $=0.65 \mathrm{~nm})$. For $\phi_{A}>60 \%$, we observed no enhancement of SAXS intensities at small $Q$ indicating that in ethanol-rich solvent all molecules are dissolved.

The two effects of oxidation mentioned above, namely, creation of insoluble/colloidal fraction in water-rich solvent and slight increase in the gyration radii of soluble molecules, are general features of all tannin oligomers (DP1-DP7). The results are summarized in Fig. 10. The radius of gyration of oxidized tannins increases with $N$ and is always slightly larger than those of nonoxidized tannins. The ratio $\alpha$ be-

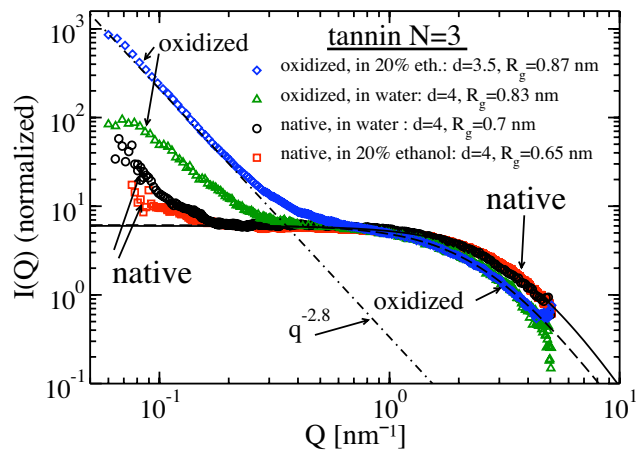

FIG. 9. (Color online) Typical effects of oxidation on native tannin trimer $(N=3)$ in solution with low ethanol content $(0 \%-20 \%)$. Scattering is ascribed to two populations: large aggregates, or T1 tannins, $\left(R_{g} \gg 10 \mathrm{~nm}\right)$ and individually soluble molecules, T2 tannins, $\left(R_{g}<1 \mathrm{~nm}\right)$. We observed no objects of intermediate size. 


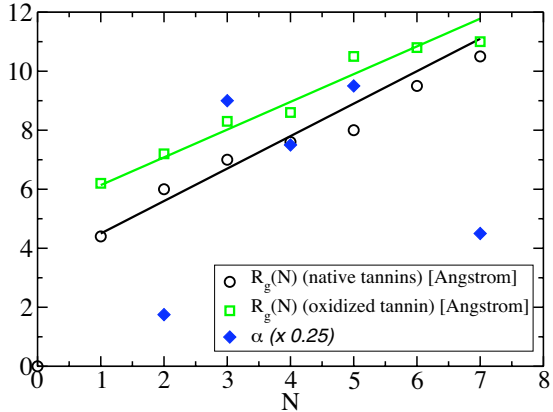

FIG. 10. (Color online) Gyration radii of oxidized and native tannin oligomers in water and the ratio $\alpha$ between low $Q$ scattering intensity from oxidized and from native tannins at $\phi_{a}=0.2$. It indicates the creation of $\mathrm{T} 1$ (colloidal) tannins by oxidation.

tween scattering intensity at lowest available momentum transfer, $Q=0.06 \mathrm{~nm}^{-1}$, from oxidized and from native tannins at $\phi_{A}=0.2$ is also shown on Fig. 10. This ratio is significantly higher than 1 (typically between 10 and 40), which signifies that species that form aggregates are created upon oxidation. These species are called T1. If the oxidation proceeds further, the low $Q$ part of the scattering profile increases even more, while high $Q$ part remains almost unchanged. Altogether, these results indicate that oxidation of tannin (1) can increase intramolecular connectivity though leaving the molecules soluble (we call this population of molecules T2) and (2) can generate the insoluble species T1 able to form colloidal particles.

Soluble fraction (T2) of oxidized tannins has similar dependence on solvent composition as native tannins. Typical changes in the shape and size of effective scatterers are of the order of $1-3 \AA$, indicating that the effect originates from the scattering on complex solvation layer, just as it was the case for native oligomers. The magnitude of the solvent effect as a function of degree of polymerizaton $N$ for both native and oxidized oligomers is shown on Fig. 7. Within experimental error, no significant difference is visible between the two sets of data.

Oxidation induces well observable changes in shapes of long tannin polymers. The effect has been studied on the case of oxidized tannin 28-mer at several solvent compositions. Data are summarized together with results for native tannin polymers on Fig. 8; structures of oxidized tannins get more extended (lower $d$ ) as $\phi_{A}$ increases, similar to the case of native molecules. At the same time, oxidized tannin molecules remain always more compact than native ones.

\section{ANALYSIS AND DISCUSSION}

Our experiments show that tannins behave in many respects as polymers in solution. From DLS results, it was concluded that the diffusivity of native tannin molecules matches with that of prolate structures, whose elongation increases with the degree of polymerization. SAXS experiments showed that at sufficiently high polymerization $N$ these structures have a fairly well defined scaling regime in scattering intensity, covering a decade of $Q$ values for tannins with $N=150$. Experiments in mixed solvent showed typical swelling effects: The better the solvent (i.e., higher the ethanol fraction $\phi_{A}$ ), the lower the scaling dimension, which varies between 2.5 in water and 1.9 in ethanol. The fact that it can be higher than 2 indicates that conformations contain a significant amount of branched structures. A transition of the scattering profile from scaling behavior to the form factor of rigid structures is expected when polymers are shorter than some characteristic correlation length, for example, Kuhn length $b$. Accordingly, the analysis of the scattering from short oligomers in mixed solvent showed that no significant variation in the size of the bare molecule and pointed mostly changes in the solvation layer. We found the Kuhn length $b=13 \pm 3 \mathrm{~nm}$. The aim of this section is to analyze in more detail and to discuss our experimental data for tannin oligomers (DP up to 7, whose length is shorter than $b$ ) and for tannin polymers (DP 28 and higher, with the size of the order and longer than $b$ ) in water-ethanol mixtures. In the light of this analysis, we interpret the experiments on oligomeric and polymeric oxidized tannin fractions.

\section{A. Tannin oligomers}

The maximal length beyond which tannin polymers can be considered as rigid rodlike structures is approximated by the Kuhn length $b$. Tannin oligomers from monomer to 7 -mer studied here are all shorter than $b$. For polymers with end-to-end length $D_{e e}$ shorter than $b$ the radius of gyration, $R_{g}$, and $D_{e e}$, can be fairly well fitted by the following expressions, linear in degree of polymerization $N$ (see Fig. 5):

$$
R_{g}(N) \approx R_{G 0}+\rho_{G}(N-1)
$$

and

$$
D_{e e}(N) \approx \eta_{e} R_{g}(N),
$$

where $R_{G 0}=0.42 \mathrm{~nm}$ is the radius of gyration of a single epicatechin, $\rho_{G}=0.1 \mathrm{~nm}$ and $\eta_{e}=3.4$. These results indicate that each epicatechin contributes to the overall length of the molecule by $\eta_{e} \rho_{G}=0.34 \mathrm{~nm}$, which corresponds approximately to the smallest width of one epicatechin. We conclude that within a tannin polymer, epicatechins are closely packed.

In order to get more detailed geometrical information about tannin oligomers, we used the program DAMMIN for reconstruction of molecular structures from SAXS data. The resulting 3D models, in Fig. 11, reveal an intriguing dumbbell shape (two-bulbs prolate) for DP4 $(N=3.8)$ and a threebulbs shape for DP7 $(N=6.3)$ polymer. We propose that those domains of larger diameter (bulbs) contain a pair of epicatechin units. Assuming that these shapes correspond to real molecular conformation and not to just a heterogeneous solvation, two interpretations are possible in terms of chemical bonds. First, short and long bonds would be C4-C8 and C4-C6. Second, all bonds would be $\mathrm{C} 4-\mathrm{C} 8$, but intermonomer distance is alternating because of alternating dihedral angle of the C4-C8 link between the closed (intrabulb) and the opened (interbulb) value. On the other hand, it is known that in apple procyanidins, studied here, $\mathrm{C} 4-\mathrm{C} 8$ bonds are dominating. Accordingly, the interpretation in terms of alternating dihedral angle is more plausible. 


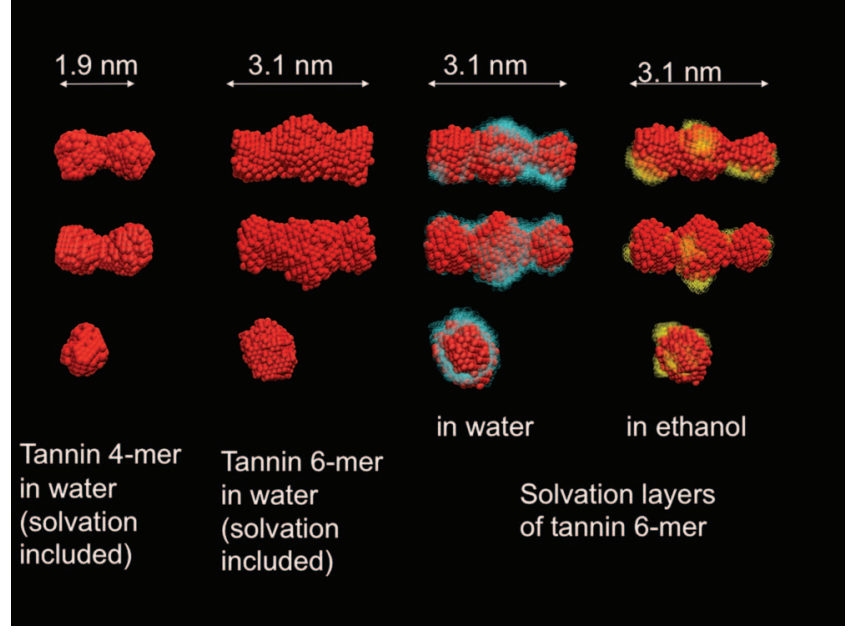

FIG. 11. (Color) Ab initio reconstructed molecular structure from the SAXS data, using DAMMIN, for tannin oligomers DP4 (average polymerization $N$ $=3.8$ ) and DP7 (average polymerization $N=6.3$ ) at a concentration of $10 \mathrm{~g} / \mathrm{l}$ in water, where shapes contain a solvation layer. Each model is shown by the front view, the view from above, and the side view. Solvation layer of (almost) dry DP7 in water and in ethanol is also shown. The side view shows that the ends of the molecules are hydrophobic: water molecules are absent.

In mixed water-ethanol solvents, SAXS patterns of oligomeric tannins depend on ethanol content. Interestingly, the apparent size, shape, and excluded volume $V_{P}$ of effective scatterers varies nonmonotonically with solvent composition, as shown in Fig. 12. A nonmonotonic dependence of polymer SAXS or SANS signal on ethanol content was recently observed and interpreted in terms of preferential solvation. ${ }^{26}$ Namely, the solvent in solvation layer has a different density and composition than the bulk solvent. This difference contributes to SAXS signal. For example, it is known that proteins in water are typically dressed by a $3 \AA$ thick hydration layer, which contributes to the contrast for $\mathrm{x}$ rays because of its density, $20 \%$ higher than the density of bulk water. ${ }^{27}$ The

DP7 $(\mathrm{N}=6.3)$ in water/ethanol solvent

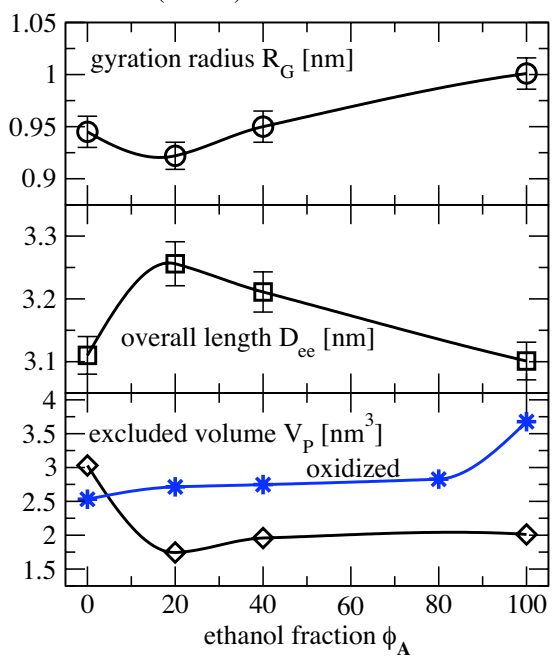

FIG. 12. (Color online) Preferential solvation effect on native DP7 tannin (average polymerization $N=6.3$ ). Radius of gyration $R_{g}$, end-to-end length $D_{e e}$, and Porod's excluded volume are nonmonotonic functions of ethanol volume fraction $\phi_{A}$. Also shown is the excluded volume of oxidized sample DP7ox monotonically increasing with $\phi_{A}$. solvation geometry in the presence of ethanol is more complicated. For example, the first maximum of the radial distribution of ethanol around a spherical apolar particle in water/ ethanol mixture is placed at about $2 \AA$ on the outside of the first hydration shell. ${ }^{28}$ In our case, this fact has yet to be combined with the heterogeneities due to amphipathic surfaces of tannins. Nevertheless, several simple results indicate that the dominant solvent effect in the case of oligomers is not the conformational change of molecules, but complex solvation around the molecules. Excluded volume $V_{P}$ remains above the estimated value of dry molecule for all values of ethanol content $\phi_{A}$. Moreover, it can be seen, on Fig. 7, that the solvent composition does not affect DP7 tannin more than it affects single monomer; variation in size and shape is about $2 \AA$ for both molecules. An important observation is that molecules have the smallest apparent size at some characteristic ethanol fraction $\phi_{A}^{B}$. For example, DP3 tannin has $\phi_{A}^{B} \approx 40 \%$. For DP7 tannin, this value is $\phi_{A}^{B}$ $\approx 20 \%$. We interpret $\phi_{A}^{B}$ as the ethanol fraction at which the solvation layer has the structure (i.e., the electronic density) closest to the bulk solvent. Therefore, the SAXS signal at $\phi_{A}=\phi_{A}^{B}$ is the closest to the hypothetic signal of a dry molecule. We identify $\phi_{A}^{B}$ as "best" or "optimal" solvent composition; we expect that solubility of tannins reaches its maximum near this value of ethanol content. Interestingly, the value $\phi_{A}^{B}$ increases as $N$ decreases: For instance, it is closer to $40 \%$ for tannin trimer. This is an indication that tannin polymers indeed fold, hiding their hydrophobic surfaces. The increasing of $\phi_{A}^{B}$ as function of $N$ is in agreement with wateroctanol partition experiment described in the Sec. II; tannins' hydrophobicity decreases with $N$ and consequently they become more soluble in water.

Polyphenols interact by two mechanisms: hydrophobic interactions by aromatic rings and hydrogen bond with solvent by phenolic $\mathrm{OH}$ functions (hydrophilic). Hydrophobic and hydrophilic sites on polyphenols molecules are disposed at the scales longer than $3 \AA$, which is comparable to the size of ethanol and water molecules. Thus, we expect complex microheterogeneity to occur around polyphenol molecules in mixed solvent. In order to explore in more detail the heterogeneous solvation of tannins, we used the differential model technique analyzing the differences between the shapes of tannins reconstructed for different solvents. As an approximation of dry tannin DP7, we took the sample in $\phi_{A}=\phi_{A}^{B}$ $\approx 20 \%$, where the Porod excluded volume is $1.74 \mathrm{~nm}^{3}$, which is only by $0.4 \mathrm{~nm}^{3}$ larger than the estimated bare volume. The 3D shape of DP7 in $\phi_{A}=\phi_{A}^{B}$ is shown in Fig. 11 . It has still a very well defined threefold structure, which confirms that multibulb structure is a characteristic of bare tannin molecule and not an effect of heterogeneous solvation. Using differential models, we found solvation layers in water and in ethanol, also shown in Fig. 11. Models indicate that solvation has heterogeneities at the scale of $1 \mathrm{~nm}$, which corresponds to the length of one tannin monomer or closely packed dimer. This confirms that solvation heterogeneities coincide with the hydropathicity pattern determined by arrangement of polyphenolic units within the polymer. 


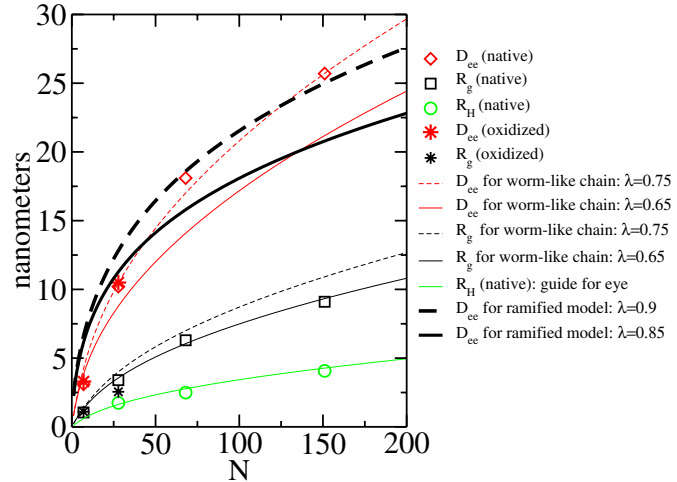

FIG. 13. (Color online) Comparison of experimental results for radius of gyration $R_{g}$ and end-to-end length $D_{e e}$ as functions of the degree of polymerization $N$, with theoretical predictions within the wormlike chain model for simple and for ramified tannins. Fitting parameters are the monomer length $a=0.8 \mathrm{~nm}$ and $\lambda$. Results for hydrodynamic radius obtained by DLS are also shown

\section{B. Tannin polymers}

For simplicity, let us first assume that in the lowest approximation ramifications can be neglected. In this case, in order to determine the Kuhn length of tannin polymers we compared our results with the wormlike chain model. ${ }^{29}$ The configuration of a chainlike polymer is usually parametrized by a set of connectivity vectors $\mathbf{X}_{i}$, with $i$ labeling the bonds between monomer $i$ and $i+1$ in the polymer sequence. The basic assumption of the wormlike chain model is that the correlation between $\mathbf{X}_{j}$ and $\mathbf{X}_{k}$ in the polymer sequence has a power-law decay,

$$
\left\langle\mathbf{X}_{j} \mathbf{X}_{k}\right\rangle=a^{2} \lambda^{|j-k|},
$$

where $a$ is a monomer length and $0<\lambda<1$ parametrizes the correlation strength. The latter can be interpreted, in the case of freely rotating chain with the valence angle $\theta$ between consecutive sites, as $\lambda=\cos \theta$. The end-to-end length $D_{e e}$ and the radius of gyration $R_{g}$ calculated within this model can be used for fitting our data. The two fitting parameters are the monomer length $a$ and the parameter $\lambda$. Radii of gyration and end-to-end length of polymers were calculated from the SAXS data. The fit to the worm-like-chain model is shown on Fig. 13. Curves are calculated by taking the monomer length $a=0.8 \mathrm{~nm}$, which is the known length of an epicatechin. The value of $\lambda$ that fits data is between 0.65 and 0.75 , corresponding to the Kuhn length $b=2 a /(1-\lambda)$ $=5.3 \pm 0.8 \mathrm{~nm}$.

In order to allow for branching in the polymer chain, the simplest worm model can be adapted as follows. The monomers that really contribute to the end-to-end length [we call their number $\tilde{N}(N)]$ are contained only in the longest possible linear chain. All other monomers belong to branches and do not contribute to the maximum end-to-end distance in the molecule. Therefore, we can still use the worm model to calculate $D_{e e}$, but instead of $N$, the corrected polymerization $\tilde{N}(N)$ must be plugged into the formula for wormlike chain of Ref. 29. From the analysis described below, we find that for long polymers $\tilde{N}$ behaves approximately as $\tilde{N} \approx 3 N^{0.6}$. Fits to experimental points for $D_{e e}$ yield values of $\lambda$ between

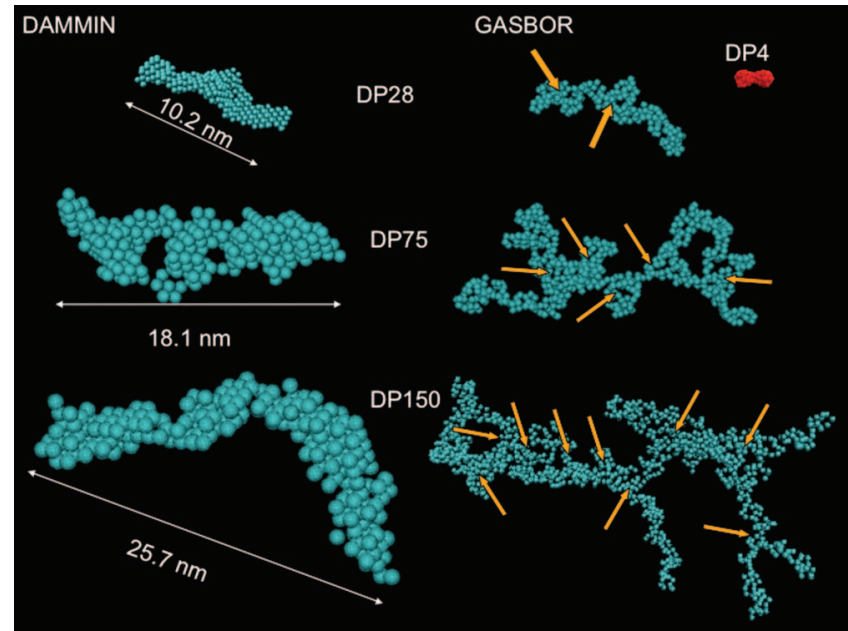

FIG. 14. (Color) Ab initio reconstructed molecular structure from the SAXS data using DAMMIN (left column) and GASBOR (right column) for tannin polymers $(10 \mathrm{~g} / \mathrm{l})$ in water with polymerization degree $N=28,75$, and 150 . Arrows show the branching sites. The DP4 model is also shown. Size scaling is the same for all models so that the proportions are real.

0.85 and 0.9 , corresponding to values of $b$ as high as 10-16 $\mathrm{nm}$. The Kuhn length is thus as long as the linear branch of about 25-43 epicatechins, or as a branched tannin with DP about 30-60.

In order to detect and quantify the branching of tannin polymers, we analyzed the SAXS patterns by using two $a b$ initio modelization strategies, implemented in DAMMIN and GASBOR programs, described in Sec. II. Both programs reconstruct molecular structures from the measured SAXS profile. Starting from a spherical structure composed of user provided number of segments, or "beads," the programs start to include cavities (dummy beads) and compare the calculated scattering profile to the experimental one. The process is iterated under specific constraints (connectivity, compactness, etc.) until the desired precision. Difference between the DAMMIN and GASBOR programs is precisely in these constraints. DAMMIN looks for the most compact bead structure, whereas GASBOR imitates "chainlike" behavior for the model. It does not put significant penalties for the looseness and requires certain number of neighbor "dummy residues" around each bead. That is why DAMMIN is not able to find starlike structures, but this is possible with GASBOR.

Figure 14 shows molecular models obtained from SAXS patterns of tannins in water. Polymers $N=28,75$, and 150 are shown, respectively, from above. The left column shows shapes obtained by DAMMIN and right column represents structures resulting from GASBOR. DAMMIN gives more compact and thicker structures, while GASBOR models have branched structure with thin chain segments, about $1 \mathrm{~nm}$ in diameter. There is no reason a priori to believe more one procedure than the other, but the following striking result makes us choose the GASBOR series as the one which is closer to the real typical tannin structure. Figure 15 shows $N$-dependence of the molecular mass of tannins calculated in two ways: From forward scattering x-ray intensity and from excluded volume calculated from Porod's invariant. The discrepancy between the two is obvious. Molecular mass calculated from the forward intensity is between once and twice 


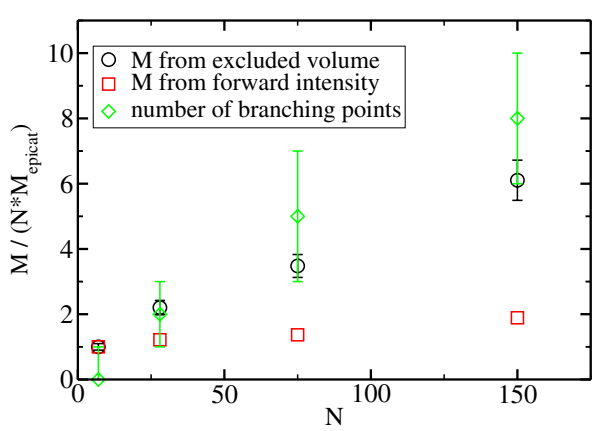

FIG. 15. (Color online) Molecular mass of tannin polymers divided by $N M_{\text {epicat }}$, where $M_{\text {epicat }}$ is the molecular mass of one epicatechin (monomer) unit. It was obtained in two independent ways: (1) from the excluded volume assuming that the structures are compact and (2) from the forward scattering intensity. It must be remembered that $N$ is the number average of the polymerization degree, measured by chemical analysis, while the SAXS takes the weight average. Due to polydispersity, the mass obtained from the SAXS forward intensity is up to twice the number average mass $N M_{\text {epicat }}$ Number of branching points (nodal monomers) as a function of $N$ is also shown.

the molecular mass expected from chemically determined polymerization degree $N$. Overestimation is due to a moderate polydispersity of the degree of polymerization: $N$ is in fact a number average, while the forward intensity measured by SAXS takes the weight average. Molecular mass calculated from the excluded volume (assuming compact packing of tannin) is much larger and cannot be explained by simple polydispersity arguments. For example, we found that the DP150 tannin has the excluded volume of $331 \mathrm{~nm}^{3}$. On the other hand, this volume can be easily estimated by knowing that one monomer (epicatechin) can be approximated by a box $0.8 \times 0.8 \times 0.3 \mathrm{~nm}^{3}$. Multiplying this volume by the polymerization degree 150 , we get the overall volume of only $29 \mathrm{~nm}^{3}$, that is 11 times smaller than Porod's excluded volume. This means that the excessive thickness of DAMMIN models is an artifact of the fitting procedure and that real structures are much less compact or much more branched. Therefore, we give more credibility to the GASBOR results. Arrows in Fig. 14 show sites at which the polymers have branching points, corresponding to epicatechins bound to three of their neighbors. The number of these nods increases with the degree of polymerization, as is also shown on Fig. 15. The molar ratio of nod monomers over total monomers is $6 \pm 1 \%$ and it is independent on the degree of polymerization.

Some caution is necessary in interpretation of molecular models obtained by GASBOR. We are aware that tannin polymeric fractions DP28, DP75, and DP150 are somewhat polydisperse in size with polydispersity index (width of the lognorm distribution) of about 0.2 , which includes both molecular mass polydispersity and also different possibilities of positions of branching sites. Furthermore, our results cannot discern if the dominant bonds within chainlike segments are $\mathrm{C} 4-\mathrm{C} 8$ or $\mathrm{C} 4-\mathrm{C} 6$ and whether the angle between subsequent monomers takes only one or both of its possible most stable values. Finally, the configurations of longer tannins can fluctuate due to their flexibility. In light of the above remarks, the GASBOR molecular model must be seen as one of possible realizations or a typical "snapshot" of tannin structure, representing the whole class of structures.

The increasing effect of branching on tannin behavior in solution, as a function of its degree of polymerization, was already pointed out by Porter. ${ }^{30}$ Our results confirm these findings and give a direct quantitative determination of the branching ratio within native tannin polymers. From the analysis of the SAXS patterns for long tannin polymers, we concluded that these molecules are branched with $6 \%$ of branching sites. It is plausible to assume that the short tannins have similar branching ratio as the long ones. For example, $6 \%$ of branching implies that one DP4 of four has a starlike structure. In our results, we found no evidence of branching in small oligomers. The reason for that might be the fact that oligomeric fractions (DP1 to DP7) were obtained from another apple variety, with ramification ratio lower than $6 \%$. This result is an indication that the value of branching ratio is not an intrinsic and general characteristic of procyanidin tannins, but is merely determined by the fruit variety and by the biological role of the tannin.

\section{Effects of controlled oxidation}

Effects of oxidation on a typical SAXS profile of a tannin is shown in Fig. 9. The formation of insoluble species is reflected by the increase in the low- $Q$ part of the scattering curve. These insoluble molecules, called T1, can aggregate via hydrophobic interactions to form colloidal particles in water rich solvent $\left(\phi_{A}<60 \%\right)$. In our experiment, the T1 fraction was of the order of $10 \%$ of total tannin mass. Nevertheless, a larger fraction of oxidized tannin remained soluble and here we shall concentrate on this soluble fraction.

Soluble fraction of oxidized tannins contributes to high- $Q$ part of the SAXS curves. The physical differences between oxidized and nonoxidized soluble tannin molecules (both called T2 for their solubility) will be discussed first for the case of a tannin oligomers on the basis of DAMMIN reconstruction. Oxidized soluble (T2) tannins are systematically larger by only a few angstroms as compared to nonoxidized ones, which confirms that oxidation did not produce any intermolecular condensation. The excluded volume $V_{P}$ of the oxidized DP7 oligomer, called DP7ox, as a function of ethanol content $\phi_{A}$ is shown on Fig. 12, together with data for native DP7. $V_{P}$ for DP7ox increases with $\phi_{A}$. Assuming that all changes in the SAXS pattern of oligomer is due to variations in the solvation layer, these data indicate that oxidized tannin prefers to be solvated by ethanol over its whole molecular surface. The homogeneous solvation of DP7ox contrasts with the heterogeneous solvation of native DP7. We thus confirm the widely recognized point that oxidation increases dramatically the hydrophobicity of tannins. We assume that the shape of dry oxidized molecules is the best represented by its apparent shape in water, where the volume of the solvation shell is minimal. The shapes of DP7ox in ethanol and in water are shown in Fig. 16. DP7ox has a similar structure as DP7 (Fig. 11), featuring three bulbs. The shape in water (approaching bare molecule shape) is $2 \AA$ longer than the native DP7 in 20\% ethanol. Their excluded volumes are also very close: DP7ox is bigger by less than 


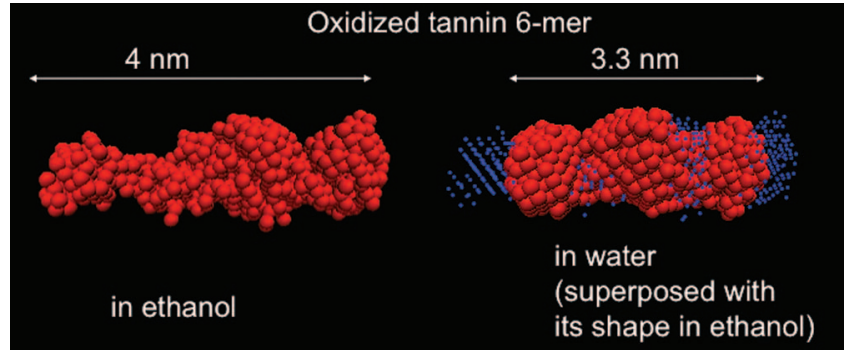

FIG. 16. (Color) Ab initio reconstructed molecular structure from the SAXS data using DAMMIN procedure for oxidized tannin oligomers DP7 (average polymerization $N=6.3$ ) in ethanol and in water. Difference between the shapes in ethanol and in water is represented by smaller beads.

$1 \mathrm{~nm}^{3}$, a difference which compares with the volume variation occurring in solvation layer. Therefore, all differences between oxidized and native oligomers can be understood in terms of differences in solvation. Our analysis shows that the pair-wise association of epicatechin units in the string of tannins is robust to oxidation. We found no qualitative differences between the overall shapes after and before oxidation. This proves that oxidation-induced links take place only between nearest neighbors and within flavane units themselves. Furthermore, the monotonic increase in the function $V_{P}\left(\phi_{A}\right)$ implies that oxidation increases hydrophobic interactions between soluble oxidized tannins.

We described higher tannin polymers (DP28 and higher) by standard polymer parameters: The Kuhn length $b$, scaling dimension $d$, and the branching ratio. Our anlaysis of oxidized DP28 tannin, called DP28ox, allowed us to find the effects of oxidation on $b$ and on solvent dependence of $d$.

Rather high polydispersity of DP28ox sample and presence of aggregates made DAMMIN analysis impossible, and therefore, the branching ratio of oxidized samples was not directly accessible. However, assuming that oxidative bonds occur only within an epicatechin itself, or between nearest neighbors within the tannin polymer, just as it was proven for $N=6$ fraction, we do not expect that the branching ratio is affected by oxidation.

The estimation of $b$ for oxidized tannins also suffers from uncertainty in the measurement due to the polydispersity and ill-defined nature of aggregates present in solution. It was possible to determine the size of DP28ox in ethanol. We found $R_{g}=5 \mathrm{~nm}$, which is slightly larger than $R_{g}$ $=4.5 \mathrm{~nm}$ of DP28. This is an indication that the Kuhn length of oxidized tannins is at least as large or even larger than those of native tannins. More precise estimation is not possible because data from higher polymers are missing. Namely, oxidation of very long tannins produces an extensively large number of new species, making samples too poydisperse.

The scaling dimension $d$ of oxidized tannin polymers DP280x as a function of $\phi_{A}$ is shown on Fig. 8(b), together with data for native polymers. For oxidized samples, $d$ is systematically higher than the $d$ of native tannins. In the same time, $d\left(\phi_{A}\right)$ is monotonically decreasing function for both native and oxidized tannins. These are indications that (1) oxidized tannins are somewhat more compact than native and (2) oxidized tannin polymers are as sensitive to solvent as native ones. This last result is somewhat surprising because one is naively tempted to assume that additional interflavane bonds make structures more rigid and therefore less sensible to solvent. However, on the other hand, the oxidized structures are more hydrophobic and, accordingly, the variation in intrachain interaction is likely more contrasted when going from water (tight hydrophobic binding) to ethanol. Thus, the final profile $d\left(\phi_{A}\right)$ is a sum of these two competing trends.

\section{SUMMARY AND CONCLUSIONS}

We investigated native and oxidized plant tannins in water/ethanol solutions by means of light scattering and SAXS. The present study focuses on homopolymers of $(-)$ epicatechin with degree of polymerization from monomer to 223-mer. We show that in water-ethanol solutions tannins are individually solvated; we found no evidence of stacking, oligomers, neither of aggregates at concentrations up to $10 \mathrm{~g} / \mathrm{l}$. SAXS results provide evidence that tannins are branched polymers and we estimated the molar ratio of trifunctional monomers to $6 \pm 1 \%$. Typical reconstructed shapes are shown in Fig. 14. The rigidity of polymers within one branch is given by the Kuhn length $b=13 \pm 3 \mathrm{~nm}$, corresponding to a linear polymer structure of 25-43 closely bound epicatechins. Tannins with overall size $D_{0}$ shorter than $b$ behave in solution like rigid linear structures. Their radius of gyration and their length are therefore linear functions of polymerization $N$ [formulas (9) and (10)]. By closer analysis of monodisperse tannin oligomers, we found that monomers are tightly bound pairwise and that these pairs are linked by longer bonds into a polymer. Typical structures of tannin oligomers are show in Fig. 11.

Effects of solvent on tannins have been studied in $\phi_{A}(v / v)$ ethanol-in-water mixtures. For tannins shorter than $b$, solvent-induced changes in SAXS are dominated by variations in composition and shape of the solvation layer. Measured size and excluded volume of solvated scatterers varies nonmonotonically with $\phi_{A}$ and these variations correspond to a solvent layer thickness of 1-3 $\AA$. These are signatures of the preferential solvation effect, a direct consequence of the amphipathic pattern of molecular surface, with well defined regions preferentially solvated by water or by ethanol. SAXS signal due to the solvation layer and the corresponding apparent size of tannin molecule reach a minimum at some finite "best solvent" composition $\phi_{A}=\phi_{A}^{B}$ (for instance, $\phi_{A}^{B}$ $=0.4$ for tannin 6-mer). Interestingly, the value of $\phi_{A}^{B}$ decreases with $N$, which means that the ratio of hydrophobic over hydrophilic surfaces depend on the degree of polymerization of oligomers. This result is in agreement with the decrease in octanol-water partition coefficient with $N$ [Fig. 2(b)].

In contrast, effects of solvent composition on tannin polymers, whose overall length is of the order or larger than $b$, are dominated by conformational changes, as indicated by the dependence of scaling dimension $d$ on ethanol content $\phi_{A}$, in Fig. 8. The monotonic decrease in $d$ with increasing $\phi_{A}$ means that tannin macromolecules swell in the presence of ethanol. This decrease in $d\left(\phi_{A}\right)$ reveals that intramolecular 
interactions are attractive and dependent of hydrogen bond. Keeping in mind that tannin polymers are very rigid within one branch, we expect that structures modify their conformation by reorientations and flexibility of the branches in nod points.

Tannin fractions were oxidized in controlled conditions. SAXS results are interpreted as in a previous work, ${ }^{7}$ in terms of water insoluble hydrophobic tannins T1 and water soluble tannins T2. Native, nonoxidized tannins belong to class T2. The first and the most evident effect of oxidation is the creation of $\mathrm{T} 1$ tannins, forming large colloidal aggregates in water rich solvents. We accordingly propose that $\mathrm{T} 1$ polyphenolic fraction in natural extracts should be composed of oxidized tannins.

Dominant fraction of oxidized tannin remains dissolved even in water rich solvent, i.e., these molecules are of class T2. The unexpected result of SAXS experiments is that these species are almost of the same size as the native ones, and, accordingly to the LC-MS analysis, almost of the same molecular mass. This result disagrees with the common assumption that oxidation of tannins promotes intermolecular condensation and increases the average molecular mass. We found the opposite: The oxidation promotes intramolecular condensations. Higher polymers, which would result from intermolecular condensation, were not detected in solution.

Interestingly, pair-polymerization shape is robust to oxidation, Fig. 16. This means that additional $\mathrm{C}-\mathrm{C}$ bonds formed upon oxidation took place only within single monomers or between first neighbors. We showed that oxidized tannins are at least as rigid as native ones and that oxidation does not affect the sensitivity of conformations to solvent composition, Fig. 8. However, at each $\phi_{A}$ oxidized tannin polymers are more compact than the native ones.

Solvation volume of oxidized tannins increases monotonically with $\phi_{A}$, which means that oxidized molecules prefer to be solvated by ethanol over a large majority of its surface, contrary to the case of native tannins. This result agrees with the well known empirical fact that oxidation increases the hydrophobic character of tannins.

\section{ACKNOWLEDGMENTS}

We thank Peter Schurtenberger for precious comments and Jean Letessier for reading the manuscript. We acknowledge the support of the European Community-Research Infrastructure Action under the FP6 "Structuring the European Research Area Programme," Contract No. RII3-CT-2004506008. D.I.S. and P.V.K. acknowledge support from the EU Design Study SAXIER, Contract No. 011934. Laboratoire de Physique Théorique et Hautes Energies is Unité Mixte de
Recherche UMR 7589 of CNRS and Université Pierre et Marie Curie-Paris 6.

${ }^{1}$ Plant Polyphenols: Synthesis, Properties, Significance, edited by R. W. Hemingway and P. E. Laks (Plenum, New York, 1992); E. Haslam, Practical Polyphenolics: From Structure to Molecular Recognition and Physiological Action, (Cambridge University Press, Cambridge, 1998).

${ }^{2}$ M. Mitek and B. Drzazga, Acta Alimentaria Polonica 14, 176 (1988).

${ }^{3}$ D. Zanchi, C. Poulain, P. Konarev, C. Tribet, and D. I. Svergun, J. Phys.: Condens. Matter 20, 494224 (2008).

${ }^{4}$ R. Gonçalves, S. Soares, N. Mateus, and V. de Freitas, J. Agric. Food Chem. 55, 7596 (2007).

${ }^{5}$ D. E. Ehrnhoefer, J. Bieschke, A. Boeddric, M. Herbst, L. Masino, R. Lurz, S. Engemann, A. Pastore, and E. E. Wanker, Nat. Struct. Mol. Biol. 15, 558 (2008).

${ }^{6}$ D. Zanchi, T. Narayanan, D. Hagenmuller, A. Baron, S. Guyot, B. Cabane, and S. Bouhallab, Europhys. Lett. 82, 58001 (2008).

${ }^{7}$ D. Zanchi, C. Poncet-Legrand, C. Tribet, R. Schweins, B. Cabane, D. Cartalade, and A. Vernhet, Langmuir 23, 9949 (2007).

${ }^{8}$ V. Cheynier, C. Prieur, S. Guyot, J. Rigaud, and M. Moutounet, ACS Symp. Ser. 661, 81 (1997).

${ }^{9}$ J. J. Macheix, J. C. Sapis, and A. Fleuriet, Crit. Rev. Food Sci. Nutr. 30, 441 (1991); J. J. Nicolas, F. C. Richard-Forget, P. M. Goupy, M. J. Amiot, and S. Y. Aubert, ibid. 34, 109 (1994).

${ }^{10}$ S. Guyot, P. Pellerin, J.-M. Brillouet, and V. Cheynier, Biosci. Biotechnol., Biochem. 60, 1131 (1996).

${ }^{11}$ L. J. Porter, in Plant Polyphenols: Synthesis, Properties, Significance, edited by R. W. Hemingway and P. E. Laks (Plenum, New York, 1992), p. 245.

${ }^{12}$ R. H. Newman, L. J. Porter, L. Y. Foo, S. R. Johns, and R. I. Willing, Magn. Reson. Chem. 25, 118 (1987).

${ }^{13}$ A. C. Fletcher, L. J. Porter, E. Haslam, and R. K. Gupta, J. Chem. Soc. [Perkin 1] 14, 1628 (1977).

${ }^{14}$ S. Guyot, N. Marnet, and J. F. Drilleau, J. Agric. Food Chem. 49, 14 (2001).

${ }^{15}$ S. Guyot (unpublished).

${ }^{16}$ C. Le Bourvellec, J.-M. Le Qur, P. Sanoner, J.-F. Drilleau, and S. Guyot, J. Agric. Food Chem. 52, 122 (2004).

${ }^{17}$ S. Bernillon, S. Guyot, and C. M. G. C. Renard, Rapid Commun. Mass Spectrom. 18, 939 (2004).

${ }^{18}$ F. Perrin, J. Phys. Radium 5, 497 (1934).

${ }^{19}$ M. W. Roessle, R. Klaering, U. Ristau, B. Robrahn, D. Jahn, T. Gehrmann, P. Konarev, A. Round, S. Fiedler, C. Hermes, and D. I. Svergun, J. Appl. Crystallogr. 40, (Supplement), s190 (2007).

${ }^{20}$ P. V. Konarev, V. V. Volkov, A. V. Sokolova, M. H. J. Koch, and D. I. Svergun, J. Appl. Crystallogr. 36, 1277 (2003).

${ }^{21}$ D. I. Svergun, J. Appl. Crystallogr. 25, 495 (1992).

${ }^{22}$ G. Porod, in General Theory, in Small-Angle X-Ray Scattering, edited by O. Glatter and O. Kratky (Academic, London, 1982), p. 17.

${ }^{23}$ M. E. Fisher and R. J. Burford, Phys. Rev. 156, 583 (1967).

${ }^{24}$ D. I. Svergun, Biophys. J. 76, 2879 (1999).

${ }^{25}$ D. I. Svergun, M. V. Petoukhov, M. H. J. Koch, Biophys. J. 80, 2946 (2001).

${ }^{26}$ B. Hammouda, J. Polym. Sci., Part B: Polym. Phys. 44, 3195 (2006).

${ }^{27}$ D. I. Svergun, S. Richard, M. H. J. Koch, Z. Sayers, S. Kuprin and G. Zaccai, Proc. Natl. Acad. Sci. U.S.A. 95, 2267 (1998).

${ }^{28}$ P. P. Shah and C. J. Roberts, J. Phys. Chem. B 111, 4467 (2007).

${ }^{29}$ P. Schurtenberger, in EmNeutrons, X-Rays and Light Scattering Methods Applied to Soft Condensed Matter, edited by P. Lindner and Th. Zemb (North Holland, Amsterdam, 2002).

${ }^{30}$ L. J. Porter, Aust. J. Chem. 39, 557 (1986). 